\title{
Collapsing Glomerulopathy Affecting Native and Transplant Kidneys in Individuals with COVID-19
}

\author{
Rebecca Noble ${ }^{a}$ Mei Ying Tan ${ }^{a}$ Thomas McCulloch ${ }^{b}$ Mohamed Shantier ${ }^{a}$ \\ Catherine Byrne $^{a}$ Matthew Hall ${ }^{a}$ Mark Jesky ${ }^{a}$ \\ aNottingham Renal and Transplant Unit, Nottingham University Hospitals NHS Trust, Nottingham, UK; \\ ${ }^{b}$ Histopathology Department, Nottingham City Hospital, Nottingham University Hospitals NHS Trust, Nottingham, UK
}

\section{Keywords}

Acute kidney injury · Collapsing glomerulopathy .

Transplant $\cdot$ Kidney biopsy $\cdot$ COVID-19

\section{Abstract}

Since the emergency of novel coronavirus COVID-19 (SARSCoV-2) in December 2019, infections have spread rapidly across the world. The reported incidence of acute kidney injury (AKI) in the context of COVID-19 is variable, and its mechanism is not well understood. Data are emerging about possible mechanisms of AKI including virus-induced cytopathic effect and cytokine storm-induced injury. To date, there have been few reports of kidney biopsy findings in the context of AKI in COVID-19 infection. This article describes 2 cases of collapsing glomerulopathy, 1 in a native kidney and, for the first time, 1 in a kidney transplant. Both individuals were black, and both presented without significant respiratory compromise. Indeed, the 2 patients we describe remained systemically well for the majority of their inpatient stay, which would support the hypothesis that for these patients, AKI was caused by a cytopathic viral effect, rather than that

karger@karger.com

(c) 2020 S. Karger AG, Basel

www.karger.com/nef

Karger" of a cytokine storm or acute tubular necrosis caused by prolonged hypovolaemia or the effect of medication known to exacerbate AKI. Here, we report 2 cases of AKI with collapsing glomerulopathy in COVID-19, one of which is in a kidney transplant recipient, not previously described elsewhere.

(c) 2020 S. Karger AG, Basel

\section{Introduction}

Since the emergence of novel coronavirus SARSCoV-2 (COVID-19) in December 2019 in Wuhan, China, the disease has spread rapidly across the world leading to the World Health Organization (WHO) declaring a worldwide pandemic in March 2020 [1]. The virus is well recognized in causing a wide range of symptoms but is most commonly associated with fever, cough, and shortness of breath with the commonest causes of mortality being acute respiratory distress syndrome and cardiovascular complications [2].

Acute kidney injury (AKI) is common in acute illness affecting 1 in 5 patients in a hospital setting [3], and its

Mark Jesky

Nottingham Renal and Transplant Unit, Nottingham University Hospitals Hucknall Road

Nottingham NG5 1PB (UK)

mark.jesky@nuh.nhs.uk 
Table 1. Parameters and biochemistry results of Patient A and Patient B

\begin{tabular}{|c|c|c|c|}
\hline Parameter & Patient A & Patient B & Reference range \\
\hline \multicolumn{4}{|l|}{ Observations on admission } \\
\hline \multicolumn{4}{|l|}{ Blood pressure, $\mathrm{mm} \mathrm{Hg}$} \\
\hline Lying & $153 / 93$ & $165 / 78$ & \multirow{3}{*}{$120 / 80$} \\
\hline Standing & NA & $135 / 58$ & \\
\hline (Lowest during admission) & $122 / 76$ & $85 / 53$ & \\
\hline Heart rate (beats per minute) & 92 & 89 & $60-100$ \\
\hline $\begin{array}{l}\text { Oxygen saturations (\%) } \\
\text { (lowest during admission) }\end{array}$ & $\begin{array}{l}96 \% \text { on room air } \\
(93 \% \text { on room air) }\end{array}$ & $\begin{array}{l}95 \% \text { on room air } \\
\text { (93\% on } 35 \% \text { oxygen) }\end{array}$ & $94-98$ \\
\hline Temperature, ${ }^{\circ} \mathrm{C}$ & 39.5 & 36.7 & $35-37.4$ \\
\hline Urine output (mL/24 h) & 90 & $157++$ (inaccurate charts) & \\
\hline (lowest pre-dialysis) & $(0)$ & (inaccurate charts) & \\
\hline \multicolumn{4}{|l|}{ Biochemistry } \\
\hline \multicolumn{4}{|l|}{$\mathrm{Cr}, \mu \mathrm{mol} / \mathrm{L}$} \\
\hline Baseline & 125 & 283 & \multirow[t]{4}{*}{$59-104$} \\
\hline Admission & 576 & 908 & \\
\hline Peak pre-dialysis & 1,202 & 1,242 & \\
\hline On discharge & 721 & Ongoing haemodialysis & \\
\hline Peak C-reactive protein, $\mathrm{mg} / \mathrm{L}$ & 157 & 308 & \multirow[t]{2}{*}{$0-10$} \\
\hline Urinalysis on admission & $3+$ blood, $4+$ protein & $3+$ blood, $3+$ protein & \\
\hline \multicolumn{4}{|l|}{ Urine PCR, mg/mmol } \\
\hline Baseline & NA & 216 & \multirow[t]{2}{*}{$<30$} \\
\hline Admission & 359 & 1,094 & \\
\hline
\end{tabular}

association with COVID-19 infections is being increasingly recognized and studied. The reported incidence of AKI in the context of COVID-19 is variable, depending on the population and setting evaluated, with quoted figures of between 0.9 and 29\% [4]. There is also evidence of new onset proteinuria and/or haematuria in individuals with COVID-19. Data are emerging about the possible mechanisms of AKI in COVID-19 and include a virusinduced cytopathic effect and cytokine storm-induced injury $[5,6]$.

Kidney transplant recipients (KTRs) are considered 1 of the groups at greatest risk for severe COVID-19 illness. Public health initiatives exist in many countries, designed to protect these high-risk groups by encouraging them to stay at home and avoid face-to-face contact (known as shielding). However, infections still occur. National and international consensus guidelines mainly focus on the respiratory impact of COVID-19, often encouraging minimizing direct patient contact if possible. To date, published reports on the impact of COVID-19 on KTRs have been descriptive [7], and, to our knowledge, there has been no report of kidney biopsy findings in a KTR. We present 2 cases of COVID-19-related AKI; both required kidney replacement therapy but no respiratory support in an intensive care unit setting.

\section{Patient A}

A 54-year-old black male presented with a past medical history of hypertension on a single antihypertensive agent (calcium channel blocker), obesity (BMI $33 \mathrm{~kg} / \mathrm{m}^{2}$ ) CKD stage G2 (baseline eGFR $65 \mathrm{~mL} / \mathrm{min}$ and Cr 125 $\mu \mathrm{mol} / \mathrm{L}$ ), and no smoking history. A week prior to admission, his primary care physician discontinued the calcium channel blocker due to pre-syncopal episodes.

He presented to the hospital complaining of productive cough, pyrexia, and reduced appetite with altered sense of taste and smell. On admission, he was haemodynamically stable and not requiring any supplemental oxygen. His parameters and biochemistry throughout his admission are listed in Table 1. Viral PCR throat swab for COVID-19 was positive, and there was no radiological evidence of COVID-19 infection on the chest X-ray. Serum Cr was 576 $\mu \mathrm{mol} / \mathrm{L}$ (AKI stage 3 by KDIGO criteria [8]). Urinalysis demonstrated haematuria and proteinuria $(3+$ blood and $4+$ protein) with urine protein:Cr ratio of $359 \mathrm{mg} / \mathrm{mmol}$.

He was initially managed with intravenous (IV) antibiotics and crystalloid fluids. As his kidney function continued to deteriorate, he was transferred to the tertiary hospital under the care of our nephrology team on day 2 of his admission. 

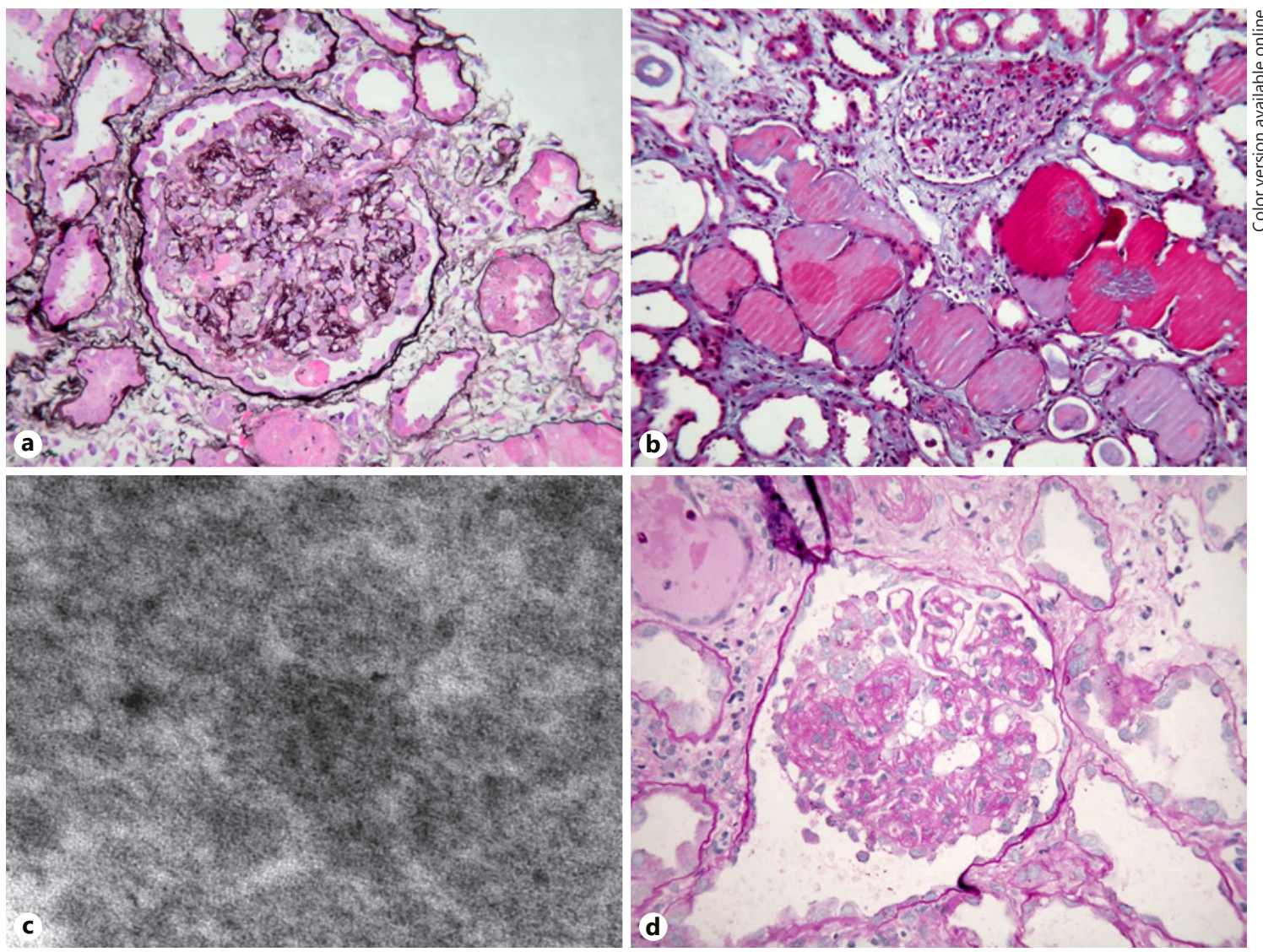

Fig. 1. Renal biopsy findings: a Patient A showing glomerular collapse with overlying tuft of hypertrophied podocytes (methanamine silver stain - original magnification $\times 200$ ). b Patient A showing tubulointerstitial features with tubular injury and dilatation and accumulation of proteinaceous fluid (trichrome original magnification $\times 200$ ). c Patient A, electron microscopy with viral-like particle in glomerular subendothelial space (original magnification $\times 30,000)$. d Patient B showing glomerular collapse (PAS original magnification $\times 200$ ).

A chest X-ray on day 3 demonstrated radiological evidence consistent with COVID-19 infection and an element of fluid overload. Serum Cr peaked at 1,202 $\mu \mathrm{mol} / \mathrm{L}$ on day 4 prior to dialysis with oliguria ( $24 \mathrm{~h}$ urine output $177 \mathrm{~mL}$ ). On ultrasonography, both kidneys demonstrated good corticomedullary differentiation and no evidence of hydronephrosis. Serology associated with glomerulonephritis was negative and serum complement $\mathrm{C} 3$ and $\mathrm{C} 4$ were normal. Antibody tests for HIV, hepatitis B and hepatitis $\mathrm{C}$, were negative. He was commenced on intermittent haemodialysis, receiving 5 sessions in total between day 4 and 16. Kidney biopsy was performed on day 19 of admission.

\section{Histopathology}

Histology of the kidney biopsy (Fig. 1) demonstrated 20 glomeruli, with at least 8 showing features of widespread and severe glomerular collapse, some global and some segmental. Collapse was demonstrable on silver staining and was often accompanied by an overlying cap of hypertrophied podocytes, some of which contained protein resorption droplets. Five of the glomeruli were globally sclerosed, attributed to pre-existing ischaemic nephropathy.

The tubulointerstitial component demonstrated severe acute tubular injury with epithelial flattening, loss of brush border, and in some areas bare tubular basement membrane. There was marked dilatation and accumulation of variably staining proteinaceous fluid in many of the tubules. Focally, tubular profiles with significant protein resorption droplets were noted. The interstitium was expanded by oedema with very little pre-existing fibrosis.

Immunofluorescence microscopy showed no significant immunoreactant deposition. Electron microscopy showed no demonstrable viral particles in the podocytes, glomerular endothelial cells, or tubular epithelial cells. However, 
multiple small vesicle-like structures were seen in the subendothelial space below the glomerular basement membrane, which had occasional morphology strongly suggestive of virions, with external spikes (Fig. 1c). In addition, endothelial cells in the glomerular capillary loops contained numerous tubuloreticular bodies, a feature suggestive of high interferon levels and in keeping with viral infection. Overall, the histological features suggested a viral cytopathy and morphologically, somewhat resembled human immunodeficiency virus-associated nephropathy.

\section{Clinical Course}

He remained apyrexial with no requirements for supplemental oxygen throughout his admission. He regained dialysis-independent renal function and was discharged on day 21 of admission with a serum $\mathrm{Cr}$ of $721 \mu \mathrm{mol} / \mathrm{L}$. At time of submission, his kidney function remained significantly impaired $(\mathrm{Cr} 715 \mu \mathrm{mol} / \mathrm{L}) 26$ days after initial presentation.

\section{Patient B}

A 45-year-old black male with type 2 diabetes mellitus requiring insulin, obesity (BMI $42.6 \mathrm{~kg} / \mathrm{m}^{2}$ ), end-stage kidney disease secondary to malignant hypertension (native kidney biopsy August 2015 - severe hypertensive changes and chronic damage), and no smoking history. He received a living donor kidney transplant in February 2016 at another transplant centre in the UK. Prior to this presentation, baseline kidney transplant function was eGFR $23 \mathrm{~mL} / \mathrm{min}(\mathrm{CKD} \mathrm{G} 4$; Cr $283 \mu \mathrm{mol} / \mathrm{L})$ and urine protein:Cr ratio $216 \mathrm{mg} / \mathrm{mmol}$.

Prescribed medication comprised tacrolimus (Adoport, Sandoz Ltd., Camberley, UK) 4 mg daily, prednisolone $5 \mathrm{mg}$ daily, mycophenolate mofetil (MMF) $3 \mathrm{~g}$ daily, 4 antihypertensive agents (bisoprolol $10 \mathrm{mg}$ daily, doxazosin $16 \mathrm{mg}$ daily, moxonidine $600 \mu \mathrm{g}$ daily, and ramipril $10 \mathrm{mg}$ daily); aspirin $75 \mathrm{mg}$ daily, and calcium/vitamin D. His diabetes was managed with NovoMix 50 insulin and liraglutide.

He was initially admitted following a collapse and found to have postural hypotension; blood pressure $165 / 78 \mathrm{~mm} \mathrm{Hg}$ (supine) to 135/58 mm Hg (standing). He was apyrexial and did not require any supplemental oxygen (Table 1). Blood tests demonstrated AKI 1 (serum Cr $413 \mu \mathrm{mol} / \mathrm{L}$ ). Chest X-ray on admission was consistent with COVID-19 infection and viral PCR throat swab was positive for COVID-19. He received IV crystalloid fluids, his antihypertensive agents were reduced, antiprolifera- tive (MMF) was omitted, and prednisolone was doubled. He was discharged on day 4 with a falling serum $\mathrm{Cr}$ of 392 $\mu \mathrm{mol} / \mathrm{L}$ and planned outpatient follow-up.

After initial discharge, he reported feeling lethargic with reduced appetite and was readmitted 6 days later, day 10 since his positive COVID-19 swab, with shock hypotension (85/53 $\mathrm{mm} \mathrm{Hg}$ ), tachycardia $125 \mathrm{bpm}$, and supplemental oxygen requirement of $4 \mathrm{~L} / \mathrm{min}$ via nasal speculae to maintain $94 \%$ oxygen saturation. He remained apyrexial. Repeat blood tests demonstrated further deterioration of kidney function (AKI 3; serum $\mathrm{Cr}$ $908 \mu \mathrm{mol} / \mathrm{L}$ ) with oliguria. Repeat chest X-ray showed progressive patchy consolidation in both lungs in keeping with COVID-19 infection. Initial management included IV crystalloid fluid resuscitation, antibiotics for hospitalacquired pneumonia with a further doubling of prednisolone to $20 \mathrm{mg}$ a day, while continuing to withhold MMF. Blood pressure improved to $110 / 74 \mathrm{~mm} \mathrm{Hg}$ within $4 \mathrm{~h}$.

He remained apyrexial and weaned off supplemental oxygen within $48 \mathrm{~h}$ of admission. His urine output improved initially, but his biochemistry did not show any improvement (peak serum Cr 1,242 $\mu \mathrm{mol} / \mathrm{L}$ ). Kidney transplant ultrasound did not detect any abnormality. Virology tests including BK virus, cytomegalovirus, HIV, and donor-specific antibodies were negative. He was commenced on intermittent haemodialysis from day 12 .

\section{Histopathology}

The biopsy (Fig. 1) was remarkably similar to that described above for Patient A with the exception that in this case, there was additional evidence of interstitial fibrosis and tubular atrophy with an inflammatory interstitial infiltrate. Of 17 glomeruli seen, 7 showed collapse, global in 3 cases, and segmental in 4 . The podocytes showed marked hypertrophy with accumulation of protein resorption droplets. The tubules showed widespread tubular injury with features very similar to Patient A with dilation and accumulation of proteinaceous fluid in areas. A number of tubular profiles showed grade 1 lymphocytic tubulitis which, in the context of the other changes, was regarded as borderline for T-cell-mediated rejection. It should be noted that his original native kidney biopsy demonstrated severe hypertensive changes with severe chronic damage. He proceeded to undergo a transplant kidney biopsy in 2017 which showed cellular rejection. Neither biopsy showed evidence of collapsing glomerulopathy.

\section{Clinical Course}

Kidney function showed no significant improvement despite a 24-h urine output of 3,000 mL. MMF was re- 
Table 2. Key teaching points

AKI is not exclusively seen in the context of severe respiratory compromise and/or a critical care setting

COVID-19 can cause a collapsing glomerulopathy most likely due to its direct cytopathic effect, including in KTRs. Cases described to date involve all individuals of black race. Further work is required to establish the relationship to this and high-risk APOL-1 variants

The aetiology in AKI is varied; urinalysis and diagnostic curiosity remain an essential part of the evaluation in those with COVID-19 and AKI

The true incidence of AKI in COVID-19 may never be fully appreciated as these patients may not otherwise present to hospital due to a lack of respiratory compromise

AKI, acute kidney injury; KTRs, kidney transplant recipients.

started on day 14 and prednisolone reduced to baseline dose. He was discharged on day 24 with regular haemodialysis planned as an outpatient, and regular blood tests and reviews to assess evidence of renal allograft recovery.

\section{Discussion}

To date, there have been few reports of kidney biopsy findings in the context of AKI in COVID-19 infection. This article describes 2 cases of collapsing glomerulopathy, 1 in a native kidney and, for the first time, 1 in a kidney transplant.

Prior to this publication, the largest case series of histological samples has been derived from a deceased population in China [9]; not all these patients developed AKI and, as the pathology is from post-mortem findings, it is difficult to know how generalizable the results are to a broader population group. Two case reports have described histological findings concordant to those presented here $[10,11]$, and it is noteworthy that the cases described are all in a black population with pre-existing hypertension. Collapsing glomerulopathy is a form of severe glomerular injury which, since its first description in the literature, has been found to be associated with multiple aetiologies, including viral illnesses, autoimmune conditions, malignancies, and some drug exposures [12].

In their article exploring possible mechanisms for AKI in COVID-19, Pan and colleagues note that the transmembrane protease serine 2 gene, one of the most important mediators of SARS-CoV-2 entering host cells was found to be co-expressed with angiotensin-converting enzyme 2 (ACE2) in podocytes [13]. This finding offers an explanation to the findings seen on electron microscopy of viral inclusion bodies in Figure 1 and in Kissling and colleagues' case report [11], and would support this

Collapsing Glomerulopathy Causing AKI in COVID-19 as a potential mechanism for collapsing glomerulopathy in COVID-19.

Why may individuals of certain ethnic groups be at higher risk of developing AKI in the context of COVID-19? Individuals with apolipoprotein L-1 (APOL-1) risk alleles are at an increased risk of progressive kidney disease, and these high-risk variants are predominantly found in individuals of African ancestry. Work investigating the many-fold higher risk of human immunodeficiency virus-associated nephropathy (a form of collapsing glomerulopathy) in individuals with $A P O L-1$ genetic variants supports a view that $A P O L-1$-mediated kidney disease follows a two-hit model of disease development [14]. Although we do not know the APOL-1 status of these individuals, one may hypothesize that the direct cytopathic infection provides the second $h i t$ and those with high-risk variants. Polymorphisms in ACE2 between ethnic groups may also have implications for the pattern of disease seen with COVID-19 infection, although this remains speculative at present $[15,16]$. Indeed, in $\mathrm{Wu}$ et al.'s [17] genetic analysis of 6 black patients with AKI and collapsing glomerulopathy associated with COVID-19, all patients had an APOL-1 high-risk genotype.

The 2 patients we describe remained systemically well without significant respiratory compromise for the majority of their inpatient stay, which would support the hypothesis that for these patients, AKI was caused by a cytopathic viral effect, rather than that of a cytokine storm or acute tubular necrosis caused by prolonged hypovolaemia or the effects of medication known to exacerbate AKI. We acknowledge that even a brief period of hypotension may have been a risk factor for AKI development in both of these patients; however, we would have expected this to have resolved with adequate fluid resuscitation. Given the strategy employed by the UK and other countries, of self-isolating if individuals develop symptoms, we are concerned there may 
be people who have developed a significant $\mathrm{AKI}$ and/or glomerulopathy but have not sought medical attention.

In summary, we have described 2 cases of AKI with collapsing glomerulopathy on kidney biopsy in patients who did not present with respiratory compromise or require management in the intensive care unit. We believe this is the first publication to describe histology of AKI in COVID-19 in a KTR. Ongoing follow-up and initiatives to share data and outcomes, such as the scheme pioneered by UK transplant registry held by Organ Donation and Transplantation, will help provide additional information in this high-risk group (Table 2).

\section{Statement of Ethics}

Patients included in this article have given written consent for their cases to be published.

\section{Conflict of Interest Statement}

The authors have no conflicts of interest to declare.

\section{Funding Sources}

No funding was obtained in the writing of this article.

\section{Author Contributions}

R.N.: data collection, drafting manuscript, revision of manuscript. M.Y.T.: data collection and drafting manuscript. T.M.: data analysis, drafting manuscript, and revision of manuscript. M.S.: drafting manuscript and revision of manuscript. C.B.: revision of manuscript. M.H.: drafting manuscript and revision of manuscript. M.J.: drafting manuscript and revision of manuscript.

\section{References}

1 Zhu N, Zhang D, Wang W, Li X, Yang B, Song J, et al. A novel coronavirus from patients with pneumonia in China, 2019. N Engl J Med. 2020;382(8):727-33.

2 Chen $\mathrm{T}, \mathrm{Wu} \mathrm{D}$, Chen $\mathrm{H}$, Yan W, Yang D, Chen G, et al. Clinical characteristics of 113 deceased patients with coronavirus disease 2019: retrospective study. BMJ. 2020;368: m1091.

3 Susantitaphong P, Cruz DN, Cerda J, Abulfaraj M, Alqahtani F, Koulouridis I, et al. World incidence of AKI: a meta-analysis. Clin J Am Soc Nephrol. 2013;8(9):1482-93.

4 Guan WJ, Ni ZY, Hu Y, Liang WH, Ou CQ, $\mathrm{He}$ JX, et al. Clinical characteristics of coronavirus disease 2019 in China. N Engl J Med. 2020;382(18):1708-20.

5 Pan X-w, Xu D, Zhang H, Zhou W, Wang L-h, Cui X-g. Identification of a potential mechanism of acute kidney injury during the $\mathrm{CO}$ VID-19 outbreak: a study based on single-cell transcriptome analysis. Intensive Care Med. 2020;46(6):1114-6.

6 Batlle D, Soler MJ, Sparks MA, Hiremath S, South AM, Welling PA, et al. Acute kidney injury in COVID-19: emerging evidence of a distinct pathophysiology. J Am Soc Nephrol. 2020;31(7):1380-83.
7 Banerjee D, Popoola J, Shah S, Ster IC, Quan $\mathrm{V}$, Phanish M. COVID-19 infection in kidney transplant recipients. Kidney Int. 2020; S0085-2538(20):30361-6.

8 Khwaja A. KDIGO clinical practice guidelines for acute kidney injury. Nephron Clin Pract. 2012;120(4):c179-84.

9 Su H, Yang M, Wan C, Yi LX, Tang F, Zhu $\mathrm{HY}$, et al. Renal histopathological analysis of 26 postmortem findings of patients with $\mathrm{CO}$ VID-19 in China. Kidney Int. 2020;98(1): 219-27.

10 Larsen CP, Bourne TD, Wilson JD, Saqqa O, Sharshir MdA. Collapsing glomerulopathy in a patient with coronavirus disease 2019 (COVID-19). Kidney Int Rep. 2020;5(6): 935-9.

11 Kissling S, Rotman S, Gerber C, Halfon M, Lamoth F, Comte D, et al. Collapsing glomerulopathy in a COVID-19 patient. Kidney Int. 2020 Jul;98(1):228-31.

12 Albaqumi M, Soos TJ, Barisoni L, Nelson PJ. Collapsing glomerulopathy. J Am Soc Nephrol. 2006;17(10):2854-63.
13 Pan XW, Xu D, Zhang H, Zhou W, Wang LH, Cui XG. Identification of a potential mechanism of acute kidney injury during the COVID-19 outbreak: a study based on single-cell transcriptome analysis. Intensive Care Med. 2020;46(6):1114-6.

14 Abid Q, Best Rocha A, Larsen CP, Schulert G, Marsh R, Yasin S, et al. APOL1-associated collapsing focal segmental glomerulosclerosis in a patient with stimulator of interferon genes (STING)-associated vasculopathy with onset in infancy (SAVI). Am J Kidney Dis. 2020;75(2):287-90

15 Smyth LJ, Cañadas-Garre M, Cappa RC, Maxwell AP, McKnight AJ. Genetic associations between genes in the renin-angiotensinaldosterone system and renal disease: a systematic review and meta-analysis. BMJ Open. 2019;9(4):e026777.

16 Alifano M, Alifano P, Forgez P, Iannelli A. Renin-angiotensin system at the heart of COVID-19 pandemic. Biochimie. 2020;174:30-3.

$17 \mathrm{Wu} \mathrm{H}$, Larsen CP, Hernandez-Arroyo CF, Mohamed MMB, Caza T, Sharshir M, et al. AKI and collapsing glomerulopathy associated with COVID-19 and APOL 1 high-risk genotype. J Am Soc Nephrol. 2020;31(8): 1688-95. 